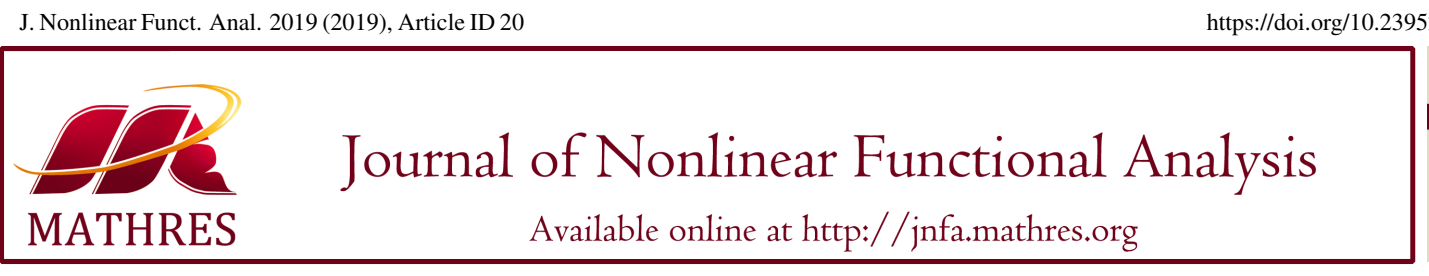

https://doi.org/10.23952/jnfa.2019.20

\title{
FIXED POINTS OF $(\Upsilon, \Lambda)$-GRAPH CONTRACTIVE MAPPINGS IN METRIC SPACES ENDOWED WITH A DIRECTED GRAPH
}

\author{
ZHENHUA MA ${ }^{1, *}$, XIANGLING LI ${ }^{1}$, ESKANDAR AMEER ${ }^{2,3}$, MUHAMMAD ARSHAD $^{3}$, SAMI ULLAH KHAN $^{4}$ \\ ${ }^{1}$ Department of Mathematics and Physics, Hebei University of Architecture, Zhangjiakou 075024, China \\ ${ }^{2}$ Department of Mathematics, Taiz University, Taiz, Yemen \\ ${ }^{3}$ Department of Mathematics, International Islamic University, H-10, Islamabad - 44000, Pakistan \\ ${ }^{4}$ Department of Mathematics, Gomal University, D. I. Khan-29050, KPK, Pakistan
}

\begin{abstract}
In this paper, we introduce a multivalued $(\Upsilon, \Lambda)$-graph contractive mapping and establish some common fixed point theorems of two multivalued mappings. An example and an application are provided to illustrate our main results.

Keywords. Directed graph; Fixed point; Metric space; Multivalued $(\Upsilon, \Lambda)$-graph contractive mapping.
\end{abstract}

2010 Mathematics Subject Classification. 47H10, 54H25.

\section{INTRODUCTION AND PRELIMINARIES}

Throughout this work, we denote by $\mathbb{R}$ the set of all real numbers, by $\mathbb{R}^{+}$the set of all positive real numbers, by $\mathbb{N}$ the set of all positive integers and by $N(X)$ the class of all nonempty subsets of $X$. It is known that the Banach contraction mapping principle is a very useful, simple and classical tool in modern analysis. Many mathematicians have investigated and generalized the Banach contraction mapping principle in different frameworks, such as, fuzzy metric spaces, $C^{*}$-algebra valued metric spaces and so on [1, 2]. In 1961, Edelstein [3] extended the well known Banach contraction principle to the fixed point theorem of uniformly locally contractive mappings in a $\varepsilon$-chainable complete metric space.

Definition 1.1. [3] A metric space $(X, d)$ is called a $\varepsilon$-chainable metric space for some $\varepsilon>0$ if, for given $x, y \in X$, there is $n \in \mathbb{N}$ and a sequence $\left\{x_{i}\right\}_{i=0}^{n}$ such that

$$
x_{0}=x, x_{n}=y \text { and } d\left(x_{i-1}, x_{i}\right)<\varepsilon, \quad \forall i=1,2, \cdots, n
$$

\footnotetext{
${ }^{*}$ Corresponding author.

E-mail addresses: mazhenghua_1981@163.com (Z. Ma),wuli67@tom.com(X. Li), eskandarameer@gmail.com (E. Ameer), marshadzia@iiu.edu.pk (M. Arshad), gomal85@gmail.com (S.U. Khan)

Received November 2, 2018; Accepted April 30, 2019.
}

(C)2019 Journal of Nonlinear Functional Analysis 
Definition 1.2. [3] Let $(X, d)$ be a complete metric space. Let $\varepsilon>0$ and $0 \leq \lambda<1$. A mapping $T: X \longrightarrow X$ is said to be $(\varepsilon, \lambda)$ uniformly locally contractive if

$$
0<d(x, y)<\varepsilon \Longrightarrow H(T(x), T(y)) \leq \lambda d(x, y), \quad \forall x, y \in X .
$$

Let $(X, d)$ be a metric space. For $x \in X$ and $A \subseteq X$, we denote by $C B(X)$ the class of all nonempty closed and bounded subsets of $X$. Let $H$ be the Hausdorff metric induced by metric $d$, that is,

$$
H(A, B)=\max \left\{\sup _{x \in A} D(x, B), \sup _{y \in B} D(y, A)\right\},
$$

where

$$
D(x, A)=\inf \{d(x, y): y \in A\} .
$$

For every $A, B \in C B(X)$, we call the metric $H$ a Pompeiu-Hausdorff metric induced by $d$. Let $T: X \longrightarrow$ $C B(X)$ be a multi-valued mapping. A point $q \in X$ is said to be a fixed point of $T$ if $q \in T q$.

In 1969, Nadler [4] gave the following multivalued version of the the famous Banach contraction principle in complete metric space.

Theorem 1.3. [4] Let $(X, d)$ be a complete metric space. Let $T: X \longrightarrow C B(X)$ be a multivalued mapping such that, for all $x, y \in X$,

$$
H(T(x), T(y)) \leq \lambda d(x, y)
$$

where $0<\lambda<1$. Then $T$ has a fixed point.

After Nadler's fixed point theorems, a number of authors obtained various multivalued fixed-point theorems; see, for example, [5, 6, 7, 8, 9, 10] and the references therein. Recently, Liu et al. [11] introduced the notion of $(\Upsilon, \Lambda)$-type Suzuki contractions and established new fixed point theorems for such kind of mappings in complete metric spaces as follows.

Definition 1.4. Let $(X, d)$ be a metric space. A mapping $T: X \longrightarrow X$ is said to be a $(\Upsilon, \Lambda)$-type Suzuki contraction if there exists a comparison function $\Upsilon$ and $\Lambda \in \Xi$ such that, for all, $x, y \in X$ with $T x \neq T y$,

$$
\frac{1}{2} d(x, T x)<d(x, y) \Longrightarrow \Lambda(d(T x, T y)) \leq \Upsilon[\Lambda(M(x, y))]
$$

where

$$
M(x, y)=\max \left\{d(x, y), d(x, T x), d(y, T y), \frac{d(x, T y)+d(y, T x)}{2}\right\},
$$

$\Xi$ is the set of functions $\Lambda:(0, \infty) \longrightarrow(0, \infty)$ satisfying the following conditions:

$(\Xi 1) \Lambda$ is non-decreasing,

( $\Xi 2)$ for each sequence $\left\{t_{n}\right\} \subset(0, \infty)$,

$$
\lim _{n \rightarrow \infty} \Lambda\left(t_{n}\right)=0 \text { if and only if } \lim _{n \rightarrow \infty} t_{n}=0,
$$

$(\Xi 3) \Lambda$ is continuous.

Recall that a function $\Upsilon:(0, \infty) \longrightarrow(0, \infty)$ is called a comparison function [12] if it satisfies the following conditions:

(1) $\Upsilon$ is monotone increasing, that is, $t_{1}<t_{2} \Longrightarrow \Upsilon\left(t_{1}\right) \leq \Upsilon\left(t_{2}\right)$,

(2) $\lim _{n \rightarrow \infty} \Upsilon^{n}(t)=0$ for all $t>0$, where $\Upsilon^{n}$ stands for the nth iterate of $\Upsilon$.

Clearly, if $\Upsilon$ is a comparison function, then $\Upsilon(t)<t$ for each $t>0$. 
Lemma 1.5. [11] Let $\Lambda:(0, \infty) \longrightarrow(0, \infty)$ be a non-decreasing and continuous function with $\inf _{t \in(0, \infty)} \Lambda\left(t_{n}\right)=$ 0 and $\left\{t_{k}\right\}_{k}$ a sequence in $(0, \infty)$. Then the following conclusion holds.

$$
\lim _{k \rightarrow \infty} \Lambda\left(t_{k}\right)=0 \text { if and only if } \lim _{k \rightarrow \infty} t_{k}=0 .
$$

Example 1.6. [12] The following functions $\Upsilon:(0, \infty) \longrightarrow(0, \infty)$ are comparison functions:

(1) $\Upsilon(t)=a t, 0<a<1$, for all $t>0$,

(2) $\Upsilon(t)=\left\{\begin{array}{lc}\frac{t}{2}, & 0<t<1, \\ \frac{t}{3}, & 1 \leq t,\end{array}\right.$

(3) $\Upsilon(t)=\frac{t}{t+1}$, for all $t>0$

Example 1.7. Define some functions as follows: for all $t \in(0, \infty)$,

(1) $\Lambda_{1}(t)=t$,

(2) $\Lambda_{2}(t)=\sqrt{t \sqrt{t}}$

(3) $\Lambda_{3}(t)=t e^{t}$,

(4) $\Lambda_{4}(t)=5^{t}$.

Then $\Lambda_{1}, \Lambda_{2}, \Lambda_{3}, \Lambda_{4} \in \Xi$.

On the other hand, we consider a directed graph $G$ such that the set of its vertices coincides with $X$ (that is, $V(G)=X$ ) and the set of its edge $E(G)=\{(x, y) \in X \times X, x \neq y\}$ and assume that $G$ has no parallel edge and weighted graph by assigning to each edge the distance between the vertices; see [13]. We can identify $G$ as $(V(G), E(G)) . G^{-1}$ denotes the conversion of a graph $G$, the graph obtained from $G$ by reversing the direction of its edges and $\widetilde{G}$ denotes the undirected graph obtained from $G$ by ignoring the direction of edge of $G$. We consider $\tilde{G}$ as a directed graph for which the set of its edges is symmetric, thus $E(\tilde{G})=E(G) \cup E\left(G^{-1}\right)$.

A subgraph of a graph $G$ is a graph $H$ such that $V(H) \subseteq V(G)$ and $E(H) \subseteq E(G)$ and for any edge $(x, y) \in E(H), x, y \in V(H)$. The number of edge in $G$ constituting the path is called the length of the path.

A graph $G$ is connected if there is a path between any two vertices of $G$. If a graph $G$ is not connected, then it is called disconnected. Moreover, $G$ is weakly connected if $\tilde{G}$ is connected. Assume that $G$ is such that $E(G)$ is symmetric and $x$ is a vertex in $G$. Then, the subgraph $G_{x}$ consisting of all edges and vertices, which are contained in some path in $G$ beginning at $x$, is called the component of $G$ containing $x$. In this case the equivalence class $[x]_{G}$ defined on $V(G)$ by the rule $R(u R v$ if there is a path from $u$ to v) is such that $V\left(G_{x}\right)=[x]_{G}$.

Let $x$ and $y$ be vertices in a graph $G$. A path in $G$ from $x$ to $y$ of length $n(n \in \mathbb{N} \cup\{0\})$ is a sequence $\left\{x_{i}\right\}_{i=0}^{n}$ of $n+1$ vertices such that $x_{0}=x, x_{n}=y$ and $\left(x_{i-1}, x_{i}\right) \in E(G)$ for $i=1,2, \ldots, n$.

Jachymski [14] introduced the following celebrated definitions.

Definition 1.8. We say that a mapping $T: X \rightarrow X$ is a Banach $G$-contraction or simply $G$-contraction if $T$ preserves edges of $G$, i.e.,

$$
\forall x, y \in X,((x, y) \in E(G) \text { implies }(T(x), T(y)) \in E(G))
$$

and $T$ decreases weights of edges of $G$ in the following way:

$$
\exists k \in(0,1), \forall x, y \in X,((x, y) \in E(G) \text { implies } d(T(x), T(y)) \leq k d(x, y)) .
$$


Definition 1.9. A mapping $T: X \rightarrow X$ is said to be $G$-continuous if, for given $x \in X$ and sequence $\left\{x_{n}\right\}$,

$$
x_{n} \rightarrow x \text { as } n \rightarrow \infty \text { and }\left(x_{n}, x_{n+1}\right) \in E(G) \text { for all } n \in N \Longrightarrow T x_{n} \rightarrow T x .
$$

Property A. [14] For any sequence $\left(x_{n}\right)_{n \in N}$ in $X$, if $x_{n} \longrightarrow x$ and $\left(x_{n}, x_{n+1}\right) \in E(G)$ for $n \in N$, then $\left(x_{n}, x\right) \in E(G)$.

Recently, Beg, Butt and Radojević [5] and Beg and Butt [6] obtained sufficient conditions for the existence of fixed points and common fixed points of multivalued graph contractive mappings in metric spaces endowed with a graph $G$.

Definition 1.10. [6] Let $(X, d)$ be a metric space endowed with a graph $G$. The mappings $S, T: X \longrightarrow$ $C B(X)$ are said to be graph contractive if there exists $k \in(0,1)$ such that

for all $x, y \in X$, with $x \neq y$ and $(x, y) \in E(G)$,

$$
H(T x, T y) \leq k d(x, y),
$$

and if $u \in T x$ and $v \in T y$ are such that $d(u, v) \leq d(x, y)$, then $(u, v) \in E(G)$.

They proved if $X_{S}=\{u \in X:(u, x) \in E(G)$, for some $x \in S u\} \neq \emptyset$, then $S, T: X \longrightarrow C B(X)$ have a common fixed point.

In this paper, we introduce a multivalued $(\Upsilon, \Lambda)$-graph contractive mapping in a metric space endowed with a directed graph. We establish common fixed point theorems of multivalued $(\Upsilon, \Lambda)$-graph contractive mappings and give examples to illustrate our main results. Common fixed points of two multivalued mappings and cyclic contraction multivalued mappings are also investigated.

The following two lemmas are useful for our main results.

Lemma 1.11. [4] Let $(X, d)$ be a metric space. If $U, V \in C B(X)$ and $u \in U$, then, for each $\varepsilon>0$, there exists $v \in V$ such that

$$
d(u, v) \leq H(U, V)+\varepsilon
$$

Lemma 1.12. [7] Let $\left\{U_{n}\right\}$ be a sequence in $C B(X)$ and $\lim _{n \rightarrow \infty} H\left(U_{n}, U\right)=0, \forall U \in C B(X)$. If $u_{n} \in U_{n}$, and $\lim _{n \rightarrow \infty} d\left(u_{n}, u\right)=0$, then $u \in U$.

\section{MAin Results}

We are now in the position to introduce the class of multivalued $(\Upsilon, \Lambda)$-graph contractive in a metric space endowed with a directed graph.

Definition 2.1. Let $(X, d)$ be a metric space endowed with a directed graph $G=(V(G), E(G))$, where $V(G)=X$. Let $S, T: X \longrightarrow C B(X)$ be two mappings. $S, T$ is said to be multivalued $(\Upsilon, \Lambda)$-graph contractive if there exist comparison function $\Upsilon$ and $\Lambda \in \Phi$ such that, for $x \neq y,(x, y) \in E(G)$,

where

$$
H(S x, T y) \neq 0 \Longrightarrow \Lambda(H(S x, T y)) \leq \Upsilon[\Lambda(M(x, y))]
$$

$$
M(x, y)=\max \left\{d(x, y), D(x, S x), \frac{D(y, S x)}{2}\right\}
$$

(b) if $u \in S x$ and $v \in T y$ and $d(u, v)<d(x, y)$, then $(u, v) \in E(G)$. 
We now prove a common fixed point theorem for multivalued $(\Upsilon, \Lambda)$-graph contractive mappings.

Theorem 2.2. Let $(X, d)$ be a complete metric space and let $G=((G), E(G))$ be a directed graph with Property A. Let $S, T: X \longrightarrow C B(X)$ be a multivalued $(\Upsilon, \Lambda)$-graph contractive and

$$
X_{S}=\{u \in X:(u, x) \in E(G), \text { for some } x \in S u\} .
$$

Then

(1) $S \backslash V\left(G_{u}\right)$ and $T \backslash V\left(G_{u}\right)$ have a common fixed point for all $u \in X_{S}$,

(2) If $G$ is weakly connected and $X_{S} \neq \emptyset$, then $S$ and $T$ have a common fixed point in $X$.

(3) If $X^{\prime}:=\cup\left\{V\left(G_{u}\right): u \in X_{S}\right\}$, then $S \backslash X^{\prime}$ and $T \backslash X^{\prime}$ have a common fixed point,

(4) If $\operatorname{Graph}(S) \subseteq E(G)$ and $E(G)$ contains all loops, then $S$ and $T$ have a common fixed point.

Proof. Conclusion (1). Letting $x_{0} \in X_{S}$, we have that there exists $x_{1} \in S x_{0}$ such that $\left(x_{0}, x_{1}\right) \in E(G)$. We can choose $n_{1} \in \mathbb{N}$ such that

$$
\left[\Upsilon\left(\Lambda\left(M\left(x_{0}, x_{1}\right)\right)\right)\right]^{n_{1}}+\Upsilon\left(\Lambda\left(M\left(x_{0}, x_{1}\right)\right)\right)<\Lambda\left(d\left(x_{0}, x_{1}\right)\right),
$$

where,

$$
M\left(x_{0}, x_{1}\right)=\max \left\{d\left(x_{0}, x_{1}\right), D\left(x_{0}, S x_{0}\right), \frac{D\left(x_{1}, S x_{0}\right)}{2}\right\},
$$

which implies from the definition of the Hausdorff metric that there exists $x_{2} \in T x_{1}$ such that

$$
\begin{aligned}
\Lambda\left(d\left(x_{1}, x_{2}\right)\right) & =\Lambda\left(d\left(x_{1}, T x_{1}\right)\right) \leq \Lambda\left(H\left(S x_{0}, T x_{1}\right)\right)+\left[\Upsilon\left(\Lambda\left(M\left(x_{0}, x_{1}\right)\right)\right)\right]^{n_{1}} \\
& \leq \Upsilon\left(\Lambda\left(M\left(x_{0}, x_{1}\right)\right)\right)+\Lambda\left(d\left(x_{0}, x_{1}\right)\right)-\Upsilon\left(\Lambda\left(M\left(x_{0}, x_{1}\right)\right)\right) \\
& <\Lambda\left(d\left(x_{0}, x_{1}\right)\right) .
\end{aligned}
$$

From (2.2) and condition ( $\Xi 1)$, we get

$$
d\left(x_{1}, x_{2}\right)<d\left(x_{0}, x_{1}\right) .
$$

Since $\left(x_{0}, x_{1}\right) \in E(G), x_{1} \in S x_{0}, x_{2} \in T x_{1}$ and $d\left(x_{1}, x_{2}\right)<d\left(x_{0}, x_{1}\right)$, we obtain from by Definition 2.1 that $\left(x_{1}, x_{2}\right) \in E(G)$.

Next, we choose $n_{2} \in \mathbb{N}$ with $n_{2}>n_{1}$ such that

$$
\left[\Upsilon\left(\Lambda\left(M\left(x_{1}, x_{2}\right)\right)\right)\right]^{n_{2}}+\Upsilon\left(\Lambda\left(M\left(x_{1}, x_{2}\right)\right)\right)<\Lambda\left(d\left(x_{1}, x_{2}\right)\right),
$$

where

$$
M\left(x_{1}, x_{2}\right)=\max \left\{d\left(x_{1}, x_{2}\right), D\left(x_{1}, S x_{1}\right), \frac{D\left(x_{2}, S x_{1}\right)}{2}\right\} .
$$

In a similar way, we see that there exists $x_{3} \in S x_{2}$ such that

$$
\begin{aligned}
\Lambda\left(d\left(x_{2}, x_{3}\right)\right) & \leq \Lambda\left(H\left(T x_{1}, S x_{2}\right)\right)+\left[\Upsilon\left(\Lambda\left(M\left(x_{1}, x_{2}\right)\right)\right)\right]^{n_{2}} \\
& \leq \Upsilon\left(\Lambda\left(M\left(x_{1}, x_{2}\right)\right)\right)+\Lambda\left(d\left(x_{1}, x_{2}\right)\right)-\Upsilon\left(\Lambda\left(M\left(x_{1}, x_{2}\right)\right)\right) \\
& <\Lambda\left(d\left(x_{1}, x_{2}\right)\right),
\end{aligned}
$$

which implies

$$
d\left(x_{2}, x_{3}\right)<d\left(x_{1}, x_{2}\right) .
$$

Since $\left(x_{1}, x_{2}\right) \in E(G), x_{2} \in T x_{1}, x_{3} \in S x_{2}$ and $d\left(x_{2}, x_{3}\right)<d\left(x_{1}, x_{2}\right)$, we obtain from Definition 2.1 that $\left(x_{2}, x_{3}\right) \in E(G)$. By induction, we obtain a sequence $\left\{x_{j}\right\}$ in $X$ and a sequence of positive integers 
$\left\{n_{j}\right\}_{j \in \mathbb{N}}$ satisfying the property that, for each $j \in \mathbb{N}, x_{2 j+1} \in S x_{2 j}$ and $x_{2 j+2} \in T x_{2 j+1},\left(x_{j}, x_{j+1}\right) \in E(G)$ and

$$
\begin{gathered}
\Lambda\left(d\left(x_{j}, x_{j+1}\right)\right)<\Lambda\left(d\left(x_{j-1}, x_{j}\right)\right), \text { for all } j \in \mathbb{N} . \\
{\left[\Upsilon\left(\Lambda\left(M\left(x_{j-1}, x_{j}\right)\right)\right)\right]^{n_{j}}+\Upsilon\left(\Lambda\left(M\left(x_{j-1}, x_{j}\right)\right)\right)<\Lambda\left(d\left(x_{j-1}, x_{j}\right)\right),}
\end{gathered}
$$

where,

$$
\begin{aligned}
& M\left(x_{j-1}, x_{j}\right)=\max \left\{d\left(x_{j-1}, x_{j}\right), D\left(x_{j-1}, S x_{j-1}\right), \frac{D\left(x_{j}, S x_{j-1}\right)}{2}\right\} \\
& \begin{aligned}
\Lambda\left(d\left(x_{j}, x_{j+1}\right)\right) & \leq \Lambda\left(H\left(T x_{j-1}, S x_{j}\right)\right)+\left[\Upsilon\left(\Lambda\left(M\left(x_{j-1}, x_{j}\right)\right)\right)\right]^{n_{j}} \\
& \leq \Lambda\left(d\left(x_{j}, x_{j+1}\right)\right), \text { when } j \text { is even }
\end{aligned}
\end{aligned}
$$

and

$$
\begin{aligned}
\Lambda\left(d\left(x_{j}, x_{j+1}\right)\right) & \leq \Lambda\left(H\left(S x_{j-1}, T x_{j}\right)\right)+\left[\Upsilon\left(\Lambda\left(M\left(x_{j-1}, x_{j}\right)\right)\right)\right]^{n_{j}} \\
& \leq \Lambda\left(d\left(x_{j-1}, x_{j}\right)\right), \text { when } j \text { is odd. }
\end{aligned}
$$

Taking into account $(\Xi 1)$, we get that $\left\{d\left(x_{j}, x_{j+1}\right)\right\}$ is decreasing and $\left(x_{j}, x_{j+1}\right) \in E(G)$. For the case that $j$ is odd, we get from (2.1) that

$$
\begin{aligned}
0 & <\Lambda\left(d\left(x_{j}, x_{j+1}\right)\right) \leq \Lambda\left(H\left(S x_{j-1}, T x_{j}\right)\right) \\
& \leq \Upsilon\left(\Lambda\left(M\left(x_{j-1}, x_{j}\right)\right)\right)
\end{aligned}
$$

where

$$
\begin{aligned}
M\left(x_{j-1}, x_{j}\right) & =\max \left\{d\left(x_{j-1}, x_{j}\right), D\left(x_{j-1}, S x_{j-1}\right), \frac{D\left(x_{j}, S x_{j-1}\right)}{2 s}\right\} \\
& \leq \max \left\{d\left(x_{j-1}, x_{j}\right), d\left(x_{j-1}, x_{j}\right)\right\} \\
& \leq d\left(x_{j-1}, x_{j}\right) .
\end{aligned}
$$

It follows that

$$
\begin{aligned}
\Lambda\left(d\left(x_{j}, x_{j+1}\right)\right) & \leq \Upsilon\left(\Lambda\left(d\left(x_{j-1}, x_{j}\right)\right)\right) \\
& <\Lambda\left(d\left(x_{j-1}, x_{j}\right)\right)
\end{aligned}
$$

which further implies,

$$
\begin{aligned}
\Lambda\left(d\left(x_{j}, x_{j+1}\right)\right) & \leq \Upsilon\left(\Lambda\left(d\left(x_{j-1}, x_{j}\right)\right)\right) \\
& \leq \Upsilon^{2}\left(\Lambda\left(d\left(x_{j-2}, x_{j-1}\right)\right)\right) \\
& \leq \cdots \\
& \leq \Upsilon^{j}\left(\Lambda\left(d\left(x_{0}, x_{1}\right)\right)\right) .
\end{aligned}
$$

Letting $j \rightarrow \infty$ in the inequality above, we get

$$
0 \leq \lim _{j \rightarrow \infty} \Lambda\left(d\left(x_{j}, x_{j+1}\right)\right) \leq \lim _{j \rightarrow \infty} \Upsilon^{j}\left(\Lambda\left(d\left(x_{0}, x_{1}\right)\right)\right)=0,
$$

which implies that

$$
\lim _{j \longrightarrow \infty} \Lambda\left(d\left(x_{j}, x_{j+1}\right)\right)=0 .
$$


This together with $(\Xi 2)$ gives

$$
\lim _{j \rightarrow \infty} d\left(x_{j}, x_{j+1}\right)=0 .
$$

For the case that $j$ is even, we can obtain the result. So, we omit the proof.

Next, we prove that $\left\{x_{j}\right\}$ is a Cauchy sequence. Arguing by contradiction, we assume that there exist $\varepsilon>0$, sequence $\left\{p_{j}\right\}_{n=1}^{\infty}$ and $\left\{q_{j}\right\}_{n=1}^{\infty}$ of natural numbers such that, for all $j \in \mathbb{N}, p_{j}>q_{j}>j$ with $d\left(x_{p(j)}, x_{q(j)}\right) \geq \varepsilon$ and $d\left(x_{p(j)-1}, x_{q(j)}\right)<\varepsilon$. Therefore,

$$
\begin{aligned}
\varepsilon & \leq d\left(x_{p(j)}, x_{q(j)}\right) \\
& \leq d\left(x_{p(j)}, x_{p(j)-1}\right)+d\left(x_{p(j)-1}, x_{q(j)}\right) \\
& <\varepsilon+d\left(x_{p(j)}, x_{p(j)-1}\right) .
\end{aligned}
$$

By taking the limit as $j \rightarrow \infty$ in (2.4), we get

$$
\lim _{n \rightarrow \infty} d\left(x_{p(j)}, x_{q(j)}\right)=\varepsilon .
$$

It follows from (2.1) that

$$
\begin{aligned}
0 & <\Lambda\left(d\left(x_{p(j)+1}, x_{q(j)+1}\right)\right) \\
& \leq \Lambda\left(H\left(S x_{p(j)}, T x_{q(j)}\right)\right) \\
& \leq \Upsilon\left(\Lambda\left(M\left(x_{p(j)}, x_{q(j)}\right)\right)\right), \quad \forall j \geq j_{0},
\end{aligned}
$$

where

$$
\begin{aligned}
M\left(x_{p(j)}, x_{q(j)}\right) & =\max \left\{d\left(x_{p(j)}, x_{q(j)}\right), D\left(x_{p(j)}, S x_{p(j)}\right), \frac{D\left(x_{q(j)}, S x_{p(j)}\right)}{2}\right\} \\
& \leq \max \left\{d\left(x_{p(j)}, x_{q(j)}\right), d\left(x_{p(j)}, x_{p(j)+1}\right), \frac{d\left(x_{q(j)}, x_{p(j)+1}\right)}{2}\right\} \\
& \leq \max \left\{d\left(x_{p(j)}, x_{q(j)}\right), d\left(x_{p(j)}, x_{p(j)+1}\right), \frac{d\left(x_{q(j)}, x_{p(j)+1}\right)}{2}\right\} .
\end{aligned}
$$

Using (2.3), (2.5) and ( $\Xi 3)$, we get

$$
\begin{aligned}
\Lambda(\varepsilon) & =\Lambda\left(d\left(x_{p(j)+1}, x_{q(j)+1}\right)\right) \\
& \leq \Lambda\left(\Upsilon\left(M\left(x_{p(j)}, x_{q(j)}\right)\right)\right) \\
& =\Upsilon(\Lambda(\varepsilon))<\Lambda(\varepsilon) .
\end{aligned}
$$

This is a contradiction. Therefore $\left\{x_{j}\right\}$ is a Cauchy sequence. Since $X$ is a complete, we can assume that $\left\{x_{j}\right\}$ converges to some point $x^{*} \in X$, that is, $\lim _{j \rightarrow \infty} x_{j}=x^{*}$.

Next, we prove that $x^{*} \in S x^{*} \cap T x^{*}$. For the case that $j$ is even, we get from the Property A that there exists a positive integer $j_{0}$ such that $\left(x_{j}, x^{*}\right) \in E(G)$, for all $j \geq j_{0}$. Since $S, T$ are a generalized $\Upsilon$ - $\Lambda$-graph contractive, we have

$$
\Lambda\left(H\left(S x_{j}, T x^{*}\right)\right) \leq \Upsilon\left(\Lambda\left(M\left(x_{j}, x^{*}\right)\right)\right) \longrightarrow 0,
$$

where

$$
M\left(x_{j}, x^{*}\right)=\max \left\{\begin{array}{c}
d\left(x_{j}, x^{*}\right), d\left(x_{j}, x_{j+1}\right), \\
\frac{d\left(x_{j+1}, x_{j+1}\right)}{2}
\end{array}\right\} .
$$


Since $x_{j+1} \in S x_{j}$ and $x_{j} \longrightarrow x^{*}$, we get $x^{*} \in T x^{*}$. For the case that $j$ is odd, we have

$$
\Lambda\left(H\left(T x_{j}, S x^{*}\right)\right) \leq \Upsilon\left(\Lambda\left(M\left(x_{j}, x^{*}\right)\right)\right) \longrightarrow 0 .
$$

Since $x_{j+1} \in T x_{j}$ and $x_{j} \longrightarrow x^{*}$, we get $x^{*} \in S x^{*}$. Since $\left(x_{j}, x_{j+1}\right) \in E(G)$ for all $j \in \mathbb{N}$ and $\left(x_{j}, x^{*}\right) \in E(G)$ for all $k \geq k_{0}$, we have $\left(u_{0}, u_{1}, \ldots, u_{j}, x^{*}\right)$ is a path in $G$. So $x^{*} \in V\left(G_{x_{0}}\right)$.

Conclusion (2). Let $x_{0} \in X_{S}$. Since $G$ is weakly connected, we have $V\left(G_{x_{0}}\right)=X$. From conclusion (1), we can get that $S$ and $T$ have a common fixed point in $X$.

Conclusion (3). From Conclusion (1), we can obtain this conclusion immediately.

Conclusion (4). If $\operatorname{Graph}(S) \subseteq E(G)$, then, for each $x^{*} \in X$, there is a point $z \in S x^{*}$ such that $\left(x^{*}, z\right) \in$ $E(G)$. So $X_{S}=X$. Since $\Delta \subseteq E(G)$, it follows that $X^{\prime}=\cup\left\{V\left(G_{x^{*}}\right): x^{*} \in X_{S}\right\}=X$. From conclusion (3), we obtain that $S$ and $T$ have a common fixed point.

Example 2.3. Consider that $G=(V(G), E(G))$ is a directed graph such that $V(G)=X=\left\{\frac{1}{2^{n}}: n \in \mathbb{N}\right\} \cup$ $\{0,1,2\}$ and $d(x, y)=|x-y|$ for all $x, y \in X$ so that $(X, d)$ is a complete metric space and

$$
E(G)=\left\{(0,0),\left(0, \frac{1}{2^{n}}\right) ; n \in \mathbb{N}\right\}
$$

Let $S, T: X \longrightarrow C B(X)$ be defined by

$$
S(x)=\left\{\begin{array}{cc}
\{0\}, & \text { if } x=0, \\
\left\{0, \frac{1}{2^{n+2}}, \frac{1}{2^{n+1}}\right\}, & \text { if } x=\frac{1}{2^{n}}, n \in \mathbb{N}, \\
\{2\}, & \text { if } x=1, \\
\{1\}, & \text { if } x=2
\end{array}\right.
$$

and

$$
T(x)=\left\{\begin{array}{cc}
\{0\}, & \text { if } x=0, \\
\left\{0, \frac{1}{2^{n+3}}, \frac{1}{2^{n+2}}\right\}, & \text { if } x=\frac{1}{2^{n}}, n \in \mathbb{N} \cup\{0\}, \\
\{2\}, & \text { if } x=2 .
\end{array}\right.
$$

Consider $\Lambda(\alpha)=5^{\alpha}$ and $\Upsilon(t)=a t, \frac{2}{3} \leq a<1$, for all $t>0$. We show that $S, T: X \longrightarrow C B(X)$ is a generalized multivalued $(\Upsilon, \Lambda)$-graph contractive mapping. Let $(x, y) \in E(G)$ such that $x \neq y$. Then $(x, y)=\left(0, \frac{1}{2^{n}}\right)$ for some $n \in \mathbb{N}$. It follows that

$$
\begin{aligned}
\Lambda\left(H\left(S(0), T\left(\frac{1}{2^{n}}\right)\right)\right) & =\Lambda\left(\frac{1}{2^{n+2}}\right) \\
& <\Upsilon\left(\Lambda\left(\max \left\{d\left(0, \frac{1}{2^{n}}\right), D(0, S 0), \frac{D\left(\frac{1}{2^{n}}, S 0\right)}{2}\right\}\right)\right)
\end{aligned}
$$

and

$$
\begin{aligned}
\Lambda\left(H\left(T(0), S\left(\frac{1}{2^{n}}\right)\right)\right) & =\frac{1}{2^{n+1}} \\
& <\Upsilon\left(\Lambda\left(\max \left\{d\left(0, \frac{1}{2^{n}}\right), D(0, T(0)), \frac{D\left(\frac{1}{2^{n}}, T(0)\right)}{2}\right\}\right)\right) .
\end{aligned}
$$

Next, let $(x, y) \in E(G)$ be such that $x \neq y$. Then $(x, y)=\left(0, \frac{1}{2^{n}}\right)$ for some $n \in \mathbb{N}$. It follows that

$$
S x=S(0)=\{0\},
$$




$$
\begin{gathered}
T y=T\left(\frac{1}{2^{n}}\right)=\left\{\frac{1}{2^{n+3}}, \frac{1}{2^{n+2}}\right\}, \\
T x=S(0)=\{0\}
\end{gathered}
$$

and

$$
S y=S\left(\frac{1}{2^{n}}\right)=\left\{0, \frac{1}{2^{n+2}}, \frac{1}{2^{n+1}}\right\} .
$$

we note that if $u \in S x, v \in T y$ and $d(u, v)<d(x, y)$, then $(u, v)$ are $\left(0, \frac{1}{2^{n+3}}\right),\left(0, \frac{1}{2^{n+2}}\right)$. So, $(u, v) \in E(G)$ and if $u^{*} \in S x, v^{*} \in T y$ and $d\left(u^{*}, v^{*}\right)<d(x, y)$, then $\left(u^{*}, v^{*}\right)$ are $(0,0),\left(0, \frac{1}{2^{n+2}}\right),\left(0, \frac{1}{2^{n+1}}\right)$. So $\left(u^{*}, v^{*}\right) \in E(G)$. It follows that $S, T$ are multivalued $(\Upsilon, \Lambda)$-graph contractive. It is easy to check that $G$ has property A. Therefore all conditions of Theorem 2.2 are satisfied. For any $u \in X_{S}, S \backslash V\left(G_{u}\right)$ and $T \backslash V\left(G_{u}\right)$ have a common fixed point. We note that $X_{S}=\{x \in X:(x, u) \in E(G)$, for some $u \in S x\}=\{0\}$ while $V\left(G_{0}\right)=$ $\left\{0, \frac{1}{2^{n}}: n \in \mathbb{N}\right\}$. Moreover, $\{0\}$ is a common fixed point of $S$ and $T$.

Remark 2.4. In Theorem 2.2, the condition $X_{S}$ can be replaced by $X_{T}$, and the condition $\operatorname{Graph}(S) \subseteq E(G)$ can be replaced by $\operatorname{Graph}(T) \subseteq E(G)$.

Corollary 2.5. Let $(X, d)$ be a complete metric space and let $G=((G), E(G))$ be a directed graph with Property A. Let $S, T: X \longrightarrow C B(X)$ be such that if there exists comparison function $\Upsilon$ and $\Lambda \in \Xi$ such that, for $x \neq y,(x, y) \in E(G)$,

(a)

$$
H(S x, T y) \neq 0 \Longrightarrow \Lambda(H(S x, T y)) \leq \Upsilon[\Lambda(d(x, y))]
$$

(b) if $u \in S x, v \in T y$ and $d(u, v)<d(x, y)$, then $(u, v) \in E(G)$ and

$$
X_{S}=\{u \in X:(u, x) \in E(G), \text { for some } x \in S u\} .
$$

Then

(1) $S \backslash V\left(G_{u}\right)$ and $T \backslash V\left(G_{u}\right)$ have a common fixed point for all $u \in X_{S}$,

(2) If $G$ is weakly connected and $X_{S} \neq \emptyset$, then $S$ and $T$ have a common fixed point in $X$.

(3) If $X^{\prime}:=\cup\left\{V\left(G_{u}\right): u \in X_{S}\right\}$, then $S \backslash X^{\prime}$ and $T \backslash X^{\prime}$ have a common fixed point,

(4) If $\operatorname{Graph}(S) \subseteq E(G)$ and $E(G)$ contains all loops, then $S$ and $T$ have a common fixed point.

Remark 2.6. Letting $\Upsilon(t)=t$, and using condition $(\Xi 1)$, we get the results announced in Beg, Butt and Radojević [5] and Beg and Butt [6]

The following result can be reached from Theorem 2.2 directly.

Corollary 2.7. Let $(X, d)$ be a complete metric space and let $G=((G), E(G))$ be a directed graph with Property A. Let $S: X \longrightarrow C B(X)$ be a mapping satisfying the following condition: there exist comparison function $\Upsilon$ and $\Lambda \in \Xi$ such that, for $x \neq y,(x, y) \in E(G)$,

(a)

$$
H(S x, S y) \neq 0 \Longrightarrow \Lambda(H(S x, S y)) \leq \Upsilon\left[\Lambda\left(\max \left\{d(x, y), D(x, S x), \frac{D(y, S x)}{2}\right\}\right)\right]
$$

(b) if $u \in S x, v \in S y$ and $d(u, v)<d(x, y)$, then $(u, v) \in E(G)$, and

$$
X_{S}=\{u \in X:(u, x) \in E(G), \text { for some } x \in S u\} .
$$


Then

(1) $S \backslash V\left(G_{u}\right)$ has a fixed point for all $u \in X_{S}$,

(2) If $G$ is weakly connected and $X_{S} \neq \emptyset$, then $S$ has a fixed point in $X$,

(3) If $X^{\prime}:=\cup\left\{V\left(G_{u}\right): u \in X_{S}\right\}$, then $S \backslash X^{\prime}$ has a fixed point,

(4) If $\operatorname{Graph}(S) \subseteq E(G)$ and $E(G)$ contains all loops, then $S$ has a fixed point,

(5) if $X_{S} \neq \emptyset$, then $\operatorname{Fix}(S) \neq \emptyset$.

Proof. Putting $S=T$ in Theorem 2.2, we find conclusions (1)-(4) easily. Conclusion (5) is a direct consequence of (1).

Finally, we apply our main result (Theorem 2.2) to common fixed points of multivalued mappings and cyclic contraction multivalued mappings in complete $\varepsilon$-chainable complete metric spaces.

Theorem 2.8. Let $(X, d)$ be a complete $\varepsilon$-chainable metric space and let $S, T: X \longrightarrow C B(X)$ be a multiivalued mapping such that there exist comparison function $\Upsilon$ and $\Lambda \in \Xi$ such that

$$
0<d(x, y)<\varepsilon \Longrightarrow \Lambda(H(S x, T y)) \leq \Upsilon\left[\Lambda\left(\max \left\{d(x, y), D(x, S x), \frac{D(y, S x)}{2}\right\}\right)\right] .
$$

If there exist $u_{0} \in S x_{0}$ and $v \in S y$ such that $0<d\left(x_{0}, u_{0}\right)<\varepsilon$. Then $S$ and $T$ have a common fixed point.

Proof. We consider the graph $G$ with $V(G)=X$, and

$$
E(G)=\Delta \cup\{(x, y) \in X \times X: 0<d(x, y)<\varepsilon\} .
$$

The $\varepsilon$-chainability of $(X, d)$ gives the connectivity of $G$. If $(x, y) \in E(G)$, then

$$
\Lambda(H(S x, T y)) \leq \Upsilon\left[\Lambda\left(\max \left\{d(x, y), D(x, S x), \frac{D(y, S x)}{2}\right\}\right)\right] .
$$

Next, we let $u \in S x, v \in T y$ and $d(u, v)<d(x, y)$. As $(x, y) \in E(G)$, we have $0<d(u, v)<\varepsilon$. We note that if $x \neq y$, then $0<d(u, v)<d(x, y)<\varepsilon$. So, $(u, v) \in E(G)$. It follows that $S, T$ are multivalued $(\Upsilon, \Lambda)$-graph contractive. Also if $x_{n} \longrightarrow x$ and $d\left(x_{n}, x_{n+1}\right)<\varepsilon$ for $n \in \mathbb{N}$, then there exists a positive integer $n_{0}$ such that $d\left(x_{n}, x\right)<\varepsilon$ for all $n \geq n_{0}$. So $\left(x_{n}, x\right) \in E(G)$. Hence, $G$ has the Property A. Since there exists $u_{0} \in S x_{0}$ such that $0<d\left(x_{0}, u_{0}\right)<\varepsilon$ and $\left(x_{0}, u_{0}\right) \in E(G)$, we have $x_{0} \in X_{S}$, that is, $X_{S} \neq \emptyset$. Therefore, $S$ and $T$ have a common fixed point by Theorem 2.2. This completes the proof.

Next, we prove a common fixed point theorem of cyclic multivalued mappings.

Theorem 2.9. Let $(X, d)$ be a complete metric space and let $m$ be a positive integer. Let $\left\{A_{i}\right\}_{i=1}^{m}$ be nonempty closed subsets of $X, Y:=\cup_{i=1}^{m} A_{i}$ and $S, T: Y \longrightarrow 2^{Y}$. Assume that $\cup_{i=1}^{m} A_{i}$ is cyclic representation of $Y$ w.r.t $S, T$ and, there exist comparison function $\Upsilon$ and $\Lambda \in \Xi$ such that, $\forall x \neq y$,

$$
\Lambda(H(S x, T y)) \leq \Upsilon\left[\Lambda\left(\max \left\{d(x, y), D(x, S x), \frac{D(y, S x)}{2}\right\}\right)\right],
$$

for $x \in A_{i}, y \in A_{i+1}$ and $A_{m+1}=A_{1}$. Then $S$ and $T$ have a common fixed point.

Proof. Since $A_{i}, i \in\{1, \ldots, m\}$ is closed, we have that $(Y, d)$ is a complete metric space. We consider the graph $G$ with $V(G)=Y$, and

$$
E(G)=\Delta \cup\left\{(x, y) \in Y \times Y: x \in A_{i}, y \in A_{i+1}, A_{m+1}=A_{1}\right\} .
$$


Let $x, y \in Y$ with $x \neq y$ and $(x, y) \in E(G)$. Then $x \in A_{i}$ and $y \in A_{i+1}$ for some $i \in\{1, \ldots, m\}$. It follows that

$$
\Lambda(H(S x, T y)) \leq \Upsilon\left[\Lambda\left(\max \left\{d(x, y), D(x, S x), \frac{D(y, S x)}{2}\right\}\right)\right] .
$$

Next, we suppose that $u \in S x, v \in T y$ and $d(u, v)<d(x, y)$. Then $u \in S x \subseteq A_{i+1}$ and $v \in T y \subseteq A_{i+2}$, $(x, y) \in E(G)$. Hence, $S, T$ are multivalued $(\Upsilon, \Lambda)$-graph contraction. Assume that $\left\{x_{n}\right\}_{n \in \mathbb{N}}$ is a sequence in $Y$ with $x_{n} \longrightarrow x$ and $\left(x_{n}, x_{n+1}\right) \in E(G)$ for $n \in \mathbb{N}$. It follows that sequence $\left\{x_{n}\right\}_{n \in \mathbb{N}}$ has infinitely many terms in each $A_{i}$, so that one can easily extract a subsequence of $\left\{x_{n}\right\}$ converging to $x$. Since $A_{i}$ is closed for all $i \in\{1, \ldots, m\}$, we have that $x \in \cap_{i=1}^{m} A_{i}$. This implies by definition of $E(G)$ that $\left(x_{n}, x\right) \in E(G)$ for $n \in \mathbb{N}$. We also note $x_{0} \in Y$. Then $x_{0} \in A_{i}$, for some $i \in\{1, \ldots, m\}$ and $S x_{0} \subseteq A_{i+1}$. Choosing $v_{0} \in S x_{0}$, we have from the definition of $E(G)$ that $\left(x_{0}, v_{0}\right) \in E(G)$. This implies $Y_{S}:=\{x \in Y:(x, v) \in E(G)$, for some $v \in S x\} \neq \emptyset$. From the definition of $E(G)$, we see that $\operatorname{Graph}(S) \subseteq E(G)$ and $\operatorname{Graph}(T) \subseteq E(G)$. Using Theorem 2.2, we can conclude that $S$ and $T$ have a common fixed point in $Y$.

\section{Funding}

This paper was supported by the Scientific Research Foundation of Education Bureau of Hebei Province (Grant No. QN2016191 )and by Doctoral Fund of Hebei University of Architecture (Grant No. B201801).

\section{REFERENCES}

[1] Z. Ma, L. Jiang, H. Sun, $C^{*}$-algebra-valued metric spaces and related fixed point theorems, Fixed Point Theory Appl. 2014 (2014), Article ID 206.

[2] Z. Ma, L. Jiang, $C^{*}$-algebra-valued b-metric spaces and related fixed point theorems, Fixed Point Theory Appl. 2015 (2015), Article ID 111.

[3] M. Edelstein, An extension of Banach's contraction principle, Proc. Amer. Math. Soc. 12 (1961), 7-10.

[4] J. Nadler, Multivalued contraction mappings, Pacific J. Math. 30 (1969), 475-478.

[5] I. Beg, A.R. Butt, S. Radojević, The contraction principle for set valued mappings on a metric space with a graph, Comput. Math. Appl. 60 (2010), 1214-1219.

[6] I Beg, A.R. Butt, Fixed point of set valued graph contractive mappings, J. Inqual. Appl. 2013 (2013), Article ID 252.

[7] N.A. Assad, W.A. Kirk, Fixed point theorems for setvalued mappings of contractive type, Pac. J. Math. 43 (1972), $533-$ 562.

[8] P.R. Patle, V. Rakocevic, D.K. Patel, An alternative partial metric approach for the existence of common fixed points, Commun. Optim. Theory, 2018 (2018), Article ID 15.

[9] H.K. Pathak, N. Shahzad, A generalization of Nadler's fixed point theorem and its applications to nonconvex integral inclusions, Topology Methods Nonlinear Anal. 41 (2013), 207-227.

[10] A. Hanjing, S. Suantai, Coincidence point and fixed point theorems for a new type of $G$-contraction multivalued mappings on a metric space endowedwith a graph, Fixed Point Theory Appl. 2015 (2015), Article ID 171.

[11] X. Liu, S.S. Chang, Y. Xiao, L. Zhao, Some fixed point theorems concerning $(\Upsilon, \Lambda)$-type contraction in complete metric spaces, J. Nonlinear Sci. Appl. 9 (2016), 4127-4136.

[12] V. Berinde, Generalized Contractions and Applications, Editura Cub Press 22, Baia Mare, (1997).

[13] R. Diestel, Graph theory, Springer. New York, (2000).

[14] J. Jachymski, The contraction principle for mappings on metric space with a graph, Proc. Amer. Math. Soc. 136 (2008), 1359-1373. 